\title{
The effect of CYP3A4 genetic variants on the susceptibility to chronic obstructive pulmonary disease in the Hainan Han population
}

\section{Huifang Shi}

A Second Affiliated Hospital of Hainan Medical University

Jianguang $X u$

A second Affiliated Hospital of Hainan Medical University

Qiong Feng

University of South China

Juan Sun

Hainan General Hospital

Yixiu Yang

Hainan General Hospital

Jie Zhao

University of South China

Xiaoli Zhou

Hainan General Hospital

Huan Niu

Hainan General Hospital

Ping $\mathrm{He}$

Hainan General Hospital

Jianfang Liu

University of South China

Quanni Li ( $D$ liquanni6868@163.com )

Hainan General Hospital

Yipeng Ding ( $\nabla$ yipengding001@163.com )

Hainan General Hospital

Research article

Keywords: COPD, CYP3A4, genetic variants, risk

Posted Date: December 9th, 2019

DOI: https://doi.org/10.21203/rs.2.18448/v1 
License: (c) (i) This work is licensed under a Creative Commons Attribution 4.0 International License. Read Full License

Version of Record: A version of this preprint was published at Genomics on November 1st, 2020. See the published version at https://doi.org/10.1016/j.ygeno.2020.07.043. 


\section{Abstract}

\section{Purpose}

Genetic polymorphisms act crucial role in chronic obstructive pulmonary disease (COPD) progression. This study was designed to investigate the correlation between CYP3A4 variants and COPD risk.

\section{Methods}

We carried out a case-control study of 821 individuals (313 patients and 508 healthy subjects) to identify the correlation of CYP3A4 SNPs with COPD risk in the Hainan Han population. The association was assessed by Odds ratios (OR) and $95 \%$ confidence intervals $(\mathrm{Cl})$.

\section{Results}

Our study showed that rs4646437 polymorphism was related to an increased susceptibility to COPD (OR $=1.45,95 \% \mathrm{Cl}=1.10-1.90, p=0.008)$. Stratified analyses indicated that the rs 4646437 polymorphism was significantly associated with an increased risk of COPD in males $(\mathrm{OR}=1.95,95 \% \mathrm{Cl}=1.19-3.20, p=$ 0.008). However, rs 4646440 played a protective role in females $(\mathrm{OR}=0.54,95 \% \mathrm{Cl}=0.31-0.93, p=0.024)$. The rs4646437 SNP was identified significantly improve the risk of COPD in smoking subjects $(O R=1.67$, $95 \% \mathrm{Cl}=1.12-2.48, p=0.011$ ). While rs 4646440 had a significantly lower susceptibility to COPD with non-smoking individuals $(\mathrm{OR}=0.64,95 \% \mathrm{Cl}=0.45-0.90, p=0.010)$. Haplotype analysis revealed that $A_{\text {rs } 4646440} T_{r s 35564277}$ and $G_{\text {rs } 4646440} T_{r s 35564277}$ haplotypes of $C Y P 3 A 4$ were found to reduce the risk of COPD in non-smoking $(\mathrm{OR}=0.61,95 \% \mathrm{Cl}=0.49-0.94, p=0.024)$.

\section{Conclusion}

Our results would give some new understanding of the association between CYP3A4 gene and COPD in the Hainan Han population.

\section{Introduction}

Chronic obstructive pulmonary disease (COPD) is a frequent pulmonary disease characterized by incomplete reversible airflow limitation usually induced by significant exposure to harmful particles or gas [1]. COPD, with high prevalence and mortality, will become the third leading cause of death in the world by 2020 according to an epidemiological survey, resulting in a huge economic and social burden, which needs to be given attention to by the whole society[2]. Although previous reports have been confirmed that cigarette smoking is the major risk exposure for COPD, host factors including genetic variants, aging and abnormal lung development also make individuals susceptible to COPD[3]. A large number of studies have been shown that gene variants such as CHRNA3, CYP1A1/2, EGLN2, RAGE, CYP2A6/7, CYP2B6 and other genes were significantly associated with risk of COPD[4]. 
Cytochrome P450 3A4 (CYP3A4), together with CYP3A5, CYP3A7 and CYP3A43 are cytochrome P450 3A subfamily members and located on chromosome 7q22 [5]. Among them, CYP3A4 is the most abundant P450 isoenzyme in human liver, involves in about $50 \%$ of the oxidative pre-systemic or systemic metabolism of drugs and makes a wide range of substrate specificity[6]. In recent years, it was observed that CYP3A4 gene have higher expression in healthy and malignant lung tissue [7, 8]. Genome-wide association studies (GWAS) and some previous studies showed that genetic polymorphisms of CYPs were significantly associated with COPD susceptibility[9-13]. However, there is not any report about the relationship between CYP3A4 variants and COPD. CYP3A4, as the member of CYPs, has been demonstrated that genetic polymorphisms of CYP3A4 could significantly influence the CYP $3 A 4$ activity[14]. And CYP3A4 variants have been identified to be associated with risk of diseases, such as HIV infection, breast cancer, type 2 diabetes mellitus, prostate cancer, lung function and so on[15-20].

Thus, four SNPs (rs3735451, rs4646440, rs3556427, and rs4646437) in the CYP3A4 gene were selected and checked by databases of 1000 genomes project and the MassARRAY platform in this study. Then we tried to assess the correlation between CYP3A4 SNPs and COPD risk in the Hainan Han population. In addition, we also investigated the CYP3A4 variants on the risk of COPD in stratified subgroups including gender and smoking status with the stratification analysis. Our present work will help to improve awareness of the molecular mechanisms of COPD and provide a fresh perspective for prevention and diagnosis of COPD.

\section{Methods \& Materials}

\section{Study subjects}

In our present study, we recruited 313 unrelated Chinese COPD patients from the Hainan General Hospital. COPD was newly diagnosed in terms of the criteria established by the Global initiative for Chronic Obstructive Lung Disease (GOLD) / COPD[3]. 508 control individuals were randomly selected from the age-matched healthy population who had a routine physical examination in the same hospital. All participates must follow the exclusion criteria: 1) History of other diseases such as bronchiectasis, tuberculosis, lung tutor, pulmonary fibrosis, heart disease and other respiratory diseases. 2) Unable to perform a chest $\mathrm{X}$-ray examination and pulmonary function tests for any reason. In addition, the controls have normal pulmonary function (volume in 1 second/forced volume capacity $>0.7$ ) and no general medical diseases or genetic disorders.

Each volunteer has notified the purpose of sample collection and written informed consent was obtained from them before this study. The participants' basic characteristics such as age, gender, smoking status, body mass index (BMI) and forced expiratory volume in 1 second (FEV1) / forced volume capacity (FVC) were recorded using standardized questionnaires, are shown in Table1. All experiments were carried out depending on the protocol of Helsinki's Declaration and were approved by the Ethics Committee of the Hainan General Hospital.

\section{SNPs selection and genotyping}


Four polymorphisms (rs3735451, rs4646440, rs3556427, and rs4646437) in CYP3A4 were selected from the database of 1000 Genomes Project. Minor allele frequency (MAF) among all SNPs was $>0.05$ in the HapMap of Chinese Han Population. Genomic DNA of whole-blood samples was extracted with wholeblood genomic DNA extraction kit (GoldMag, Xi'an, China). The purity and concentration of genomic DNA were tested using NanoDrop 2000C spectrophotometer (Thermo Scientific, Waltham, USA). PCR primers in this study were designed by Agena Bioscience Assay Design Suite software (Version 2.0), and showed in Table 2. Agena MassARRAY iPLEX platform was applied for identifying SNP genotyping. The data was managed and analyzed by Agena Bioscience TYPER version 4.0 software.

\section{Statistical analysis}

We used the SPSS version 17.0 software to perform statistical analysis. All statistical tests were twotailed and $p<0.05$ indicated statistical significance. The differences in demographic and clinical characteristics between the case and control participants were analyzed by $t$-test and $\chi^{2}$ tests. HardyWeinberg equilibrium (HWE) of SNPs in controls was evaluated with the exact test. The allele and genotype distribution in cases and controls was compared with a $\chi^{2}$ test or exact test. ORs and $95 \% \mathrm{Cls}$ were used to evaluate associations between CYP3A4 variants and COPD susceptibility using logistic regression analysis with or without adjustment for age and gender. Multiple genetic models (co-dominant, dominant, recessive and log-additive) were tested by PLINK software. Stratified models were applied for subgroup analysis in male and female, smoking and non-smoking participants. Linkage disequilibrium (LD), haplotype construction and genetic associations of polymorphism loci were estimated by Haploview software (Version 4.2) and logistic regression analyses.

\section{Results}

\section{General characteristics of the study participants}

The basic characteristics of participants in our present study were listed in Table1. Total of 821 subjects were recruited including 313 patients with COPD (238 males and 75 females) and 508 healthy controls (337 males and 171 females). The average age was $71.80 \pm 10.09$ years in cases and $60.05 \pm 6.48$ years in controls. There were no significant differences on the smoking status and BMI distribution among case and control group ( $p=0.082$ and $p=0.587$, respectively).

\section{Association between CYP3A4 variants and COPD risk}

Detail information and allele frequencies of selecting SNPs are described in Table3. Four SNPs (rs3735451, rs4646440, rs35564277 and rs4646437) of the CYP3A4 gene were successfully genotyped in this study. The observed genotype frequency of rs3735451 was not in agreement with HWE in control populations $(p<0.05)$. Multiple genetic models adjusted for age and gender were analyzed for examining the relationship between SNPs and COPD risk. Our results revealed that SNP rs4646437 exhibited more significant difference than other SNPs in allele or genotype frequency between case and control individuals (Table 3, Table 4). We found that rs 4646437 polymorphism was associated with a significant 
increased susceptibility to COPD under allele model ( $\mathrm{A}$ vs $\mathrm{G}, \mathrm{OR}=1.45,95 \% \mathrm{Cl}=1.10-1.90, p=0.008$ ), codominant model ( $\mathrm{AG}$ vs GG, OR $=1.53,95 \% \mathrm{Cl}=1.02-2.28, p=0.039$ ) and dominant model (AG-AA vs GG, $\mathrm{OR}=1.49,95 \% \mathrm{Cl}=1.00-2 \cdot 20, p=0.048$ ). Another two SNPs (rs4646440 and rs35564277) were not observed a significant association with the risk of COPD.

\section{Stratification analysis}

We evaluated the association between CYP3A4 variants and COPD risk with stratified analysis according to gender and smoking status. Stratified analysis by gender indicated that the rs 4646437 polymorphism was significantly related to an increased risk of COPD in males (A vs G, OR $=1.62,95 \% \mathrm{Cl}=1.19-2.22, p=$ 0.002 ; $A G$ vs $G G, O R=1.95,95 \% \mathrm{Cl}=1.19-3.20, p=0.008$ and $A G-A A$ vs $G G, O R=1.89,95 \% \mathrm{Cl}=1.17-$ $3.05, p=0.010$, Table 5). However, rs 4646440 was a protective role in females (A vs $\mathrm{G}, \mathrm{OR}=0.54,95 \% \mathrm{Cl}$ $=0.31-0.93, p=0.024$, Table 5). We also examined the relationship of CYP3A4 SNPs and COPD risk under smoking status subgroup. As is summarized in Table 6, the rs 4646437 SNP was found to significantly improve the risk of COPD in smoking subjects ( $A$ vs $G, O R=1.67,95 \% \mathrm{Cl}=1.12-2.48, p=0.011$; $A G$ vs $G G$, $\mathrm{OR}=2.03,95 \% \mathrm{Cl}=1.02-4.04, p=0.043$ and $\mathrm{AG}-\mathrm{AA}$ vs $\mathrm{GG}, \mathrm{OR}=2.03,95 \% \mathrm{Cl}=1.03-3.99, p=0.042)$. While rs 4646440 had a significantly lower susceptibility to COPD with non-smoking individuals (A vs $G$, $\mathrm{OR}=0.64,95 \% \mathrm{Cl}=0.45-0.90, \mathrm{p}=0.010 ; \mathrm{AG}$ vs GG, OR =0.61, 95\% $\mathrm{Cl}=0.38-0.98, p=0.039$ and $\mathrm{AG}-\mathrm{AA}$ vs $\mathrm{GG}, \mathrm{OR}=0.59,95 \% \mathrm{Cl}=0.37-0.94, p=0.027)$. No significant associations observed in other SNPs.

\section{Haplotype analyses of SNPs and COPD risk}

We further studied LD and haplotype analyses for exploring the association of CYP3A4 gene with COPD risk in all subjects. LD analysis disclosed that two SNPs including rs4646440 and rs35564277 formed a LD block in non-smoking subgroup (Figure 1). Haplotype distribution between case and control group was displayed in Table 7. And the haplotype analysis indicated that $\mathrm{A}_{\mathrm{rs} 4646440} \mathrm{~T}_{\mathrm{rs} 35564277}$ and $\mathrm{G}_{\mathrm{rs} 4646440} \mathrm{~T}_{\mathrm{rs} 35564277}$ haplotypes of $C Y P 3 A 4$ were found to reduce the risk of COPD $(\mathrm{OR}=0.61,95 \% \mathrm{Cl}=$ $0.37-0.99, p=0.048 ; \mathrm{OR}=0.61,95 \% \mathrm{Cl}=0.49-0.94, p=0.024 ;$ respectively).

\section{Discussion}

In this case-control study, we investigated the association between CYP3A4 variants (rs3735451, rs4646440, rs3556427 and rs4646437) and COPD susceptibility in a Hainan Han population. We found that rs4646437 polymorphism associates with a significantly increased susceptibility to COPD.

Furthermore, rs4646437 polymorphism significantly increased risk of COPD in male and smoking individuals. While rs 4646440 showed a significant correlation with reduced susceptibility to COPD in female and non-smoking subjects. These results demonstrate that CYP3A4 polymorphisms may play a critical role in COPD risk of Hainan Han populations. As far as we know, this is the first study to test the genetic polymorphisms of CYP3A4 effecting on COPD risk in the Hainan Han population which may give some available information for the prevention and diagnosis of COPD. 
CYP3A4 was particularly considered to be an enzyme that is the basis for certain drug induction or cascade regulation of various cytokines and their downstream signals which have a high expression level in human hepatocytes[21, 22]. In recent years, increased evidence showed that genetic variability of CYP3A4 contributes significantly to lung function in humans[23]. That happens because the CYP3A4 can modulate lung inflammation, the composition of airway lining fluid and the tone of pulmonary vasculature according to catalyze the oxidative metabolism and metabolic activation of most toxicants in human lung[24]. The occurrence of COPD usually caused by significant exposure to noxious particles or gases owing to pulmonary dysfunction. These suggest that $C Y P 3 A 4$ variants may play an important role in the progression of COPD.

Genetic polymorphisms of the CYP3A4 gene can influence the protein expression level, which might associate with the occurrence of diseases. Thus, we propose that the CYP3A4 variants can affect the function of a gene resulting in the COPD progression. However, there is not any finding of correlation between CYP3A4 variants and COPD risk. In this study, we found that rs 4646437 polymorphism could dramatically increase the susceptibility to COPD in a Hainan Han population. We further examined the correlation between CYP3A4 variants and COPD risk via stratified analysis including gender and smoking status. Upon gender-based stratification, our results showed that rs 4646437 polymorphism was significantly related to an increased risk of COPD in males, while rs 4646440 played a protective role in females. Stratified by smoking status, it showed that rs 4646437 significantly improved COPD susceptibility in smoking subjects, while rs 4646440 had a significantly lower susceptibility to COPD with non-smoking individuals. These results indicated that genetic variants of CYP3A4 were associated with COPD susceptibility, and the risk association of the genetic variants may rely on the gender and smoking status. Cigarette smoking is the most commonly risk factor for COPD[25]. Tobacco smoke makes up of polycyclic aromatic hydrocarbons (PAHs)[26]. The activated PAHs catalyzed by CYP3A will reduce lung function in smokers[27, 28]. Rs4646437 and rs4646440 SNPs are located in the intron region, which might affect regulation of the CYP3A4 gene expression in COPD with smokers. Several studies supported the genetic variants confer susceptibility by affecting CYP3A4 gene expression[29, 30]. Thus, we conjectured that CYP3A4 variants, especially rs4646437 and rs4646440 may affect the CYP3A4 gene expression in the progression of COPD and need to be further explored.

Several limitations of our study are summarized as following. First, we investigated the genetic factors association with COPD susceptibility but there were not detecting gene-environment interactions in present study. Future work is needed to detect gene-environment interactions in risk of COPD. Second, we just determined role of CYP3A4 variants in risk of COPD, further research should focus on the molecular mechanism of CYP3A4 variants on COPD occurrence. Despite the above limitations, our present work provides the available evidence of the CYP3A4 gene with COPD for the future study.

\section{Conclusions}

In summary, our present work indicated that the CYP $3 A 4$ variants were associated with COPD susceptibility. Rs4646437 polymorphism were significantly associated with increasing COPD risk. 
Stratified analysis showed that rs 4646437 was significantly associated with an increased risk of COPD in males, while rs 4646440 played a protective role in female. Rs4646437 significantly improved COPD susceptibility in smoking subjects, while rs 4646440 had a significantly lower susceptibility to COPD with non-smoking individuals. In addition, the haplotype analysis indicated that $\mathrm{A}_{\mathrm{rs} 4646440} \mathrm{~T}_{\mathrm{rs} 35564277}$ and $\mathrm{G}_{\mathrm{rs} 4646440} \mathrm{~T}_{\mathrm{rs} 35564277}$ haplotypes of CYP3A4 were found to reduce the COPD risk in non-smoking. Our present study will provide a useful information for prevention and diagnosis of COPD in the future.

\section{Declarations}

\section{Ethics approval and consent to participate}

All procedures performed in studies involving human participants were in accordance with the ethical standards of the Hainan General Hospital and with the 1964 Helsinki declaration. Informed consent was obtained from all individual participants included in the study.

\section{Consent for publication}

Not applicable.

\section{Availability of data and materials}

All data generated or analysed during this study are included in this published article.

\section{Competing interests}

All authors declare that they have no competing interests.

\section{Funding}

This study was supported by the National Natural Science Foundation of China (No. 81660013 and No.81860015) and Key Research and Development Plan of Hainan Province『No. ZDYF2018116》

\section{Authors' contributions}

HF, QN, YP: Conceived and designed the experiments. JG, Q, JX, X, XZ, HN: Recruited and collected study samples. HF, XL, H: Selected the SNPs and designed primers. HF, QN: Performed the data and wrote the manuscript. HF JG, QN, YP: Contributed to data analysis and manuscript revised. All authors read and approved the final manuscript.

\section{Acknowledgements}

The authors thank all participants and volunteers in this study. We also thank the Hainan General Hospital for their helping with sample collections. 


\section{References}

1. Mirza S, Clay RD, Koslow MA, Scanlon PD: COPD Guidelines: A Review of the 2018 GOLD Report. Mayo Clinic proceedings 2018, 93(10):1488-1502.

2. Yamaguti WP, Paulin E, Salge JM, Chammas MC, Cukier A, Carvalho CR: Diaphragmatic dysfunction and mortality in patients with COPD. Jornal brasileiro de pneumologia : publicacao oficial da Sociedade Brasileira de Pneumologia e Tisilogia 2009, 35(12):1174-1181.

3. Vogelmeier CF, Criner GJ, Martinez FJ, Anzueto A, Barnes PJ, Bourbeau J, Celli BR, Chen R, Decramer M, Fabbri LM et al: Global Strategy for the Diagnosis, Management, and Prevention of Chronic Obstructive Lung Disease 2017 Report: GOLD Executive Summary. The European respiratory journal 2017, 49(3).

4. Ding $Y$, Niu H, Yang H, Sun P, Chen Y, Duan M, Xu D, Xu J, Jin T: EGLN2 and RNF150 genetic variants are associated with chronic obstructive pulmonary disease risk in the Chinese population. International journal of chronic obstructive pulmonary disease 2015, 10:145-151.

5. Werk AN, Cascorbi I: Functional gene variants of CYP3A4. Clinical pharmacology and therapeutics 2014, 96(3):340-348.

6. Zhou Q, Yu X, Shu C, Cai Y, Gong W, Wang X, Wang DM, Hu S: Analysis of CYP3A4 genetic polymorphisms in Han Chinese. Journal of human genetics 2011, 56(6):415-422.

7. Nakajima T, Elovaara E, Gonzalez FJ, Gelboin HV, Raunio H, Pelkonen O, Vainio H, Aoyama T: Styrene metabolism by cDNA-expressed human hepatic and pulmonary cytochromes P450. Chemical research in toxicology 1994, 7(6):891-896.

8. Bieche I, Narjoz C, Asselah T, Vacher S, Marcellin P, Lidereau R, Beaune P, de Waziers I: Reverse transcriptase-PCR quantification of mRNA levels from cytochrome (CYP)1, CYP2 and CYP3 families in 22 different human tissues. Pharmacogenetics and genomics 2007, 17(9):731-742.

9. Uslu A, Ogus C, Ozdemir T, Bilgen T, Tosun O, Keser I: The effect of CYP1A2 gene polymorphisms on Theophylline metabolism and chronic obstructive pulmonary disease in Turkish patients. $B M B$ reports 2010, 43(8):530-534.

10. Korytina GF, Akhmadishina LZ, Kochetova OV, Zagidullin Sh Z, Viktorova TV: [Association of cytochrome P450 genes polymorphisms (CYP1A1 and CYP1A2) with the development of chronic obstructive pulmonary disease in Bashkortostan]. Molekuliarnaia biologiia 2008, 42(1):32-41.

11. Minematsu N, Nakamura H, Iwata M, Tateno H, Nakajima T, Takahashi S, Fujishima S, Yamaguchi K: Association of CYP2A6 deletion polymorphism with smoking habit and development of pulmonary emphysema. Thorax 2003, 58(7):623-628.

12. Kaur-Knudsen D, Bojesen SE, Nordestgaard BG: CHRNA3 and CYP3A5*3 genotype, lung function and chronic obstructive pulmonary disease in the general population. Pharmacogenetics and genomics 2014, 24(4):220-229.

13. Mount S, Cirillo E, Stewart K, Coort S, Evelo CT, Wesselius A, Zeegers MP, Schols A: Network Analysis of Genome-Wide Association Studies for COPD in the Context of Biological Pathways. American 
journal of respiratory and critical care medicine 2019.

14. Lutz SM, Frederiksen B, Begum F, McDonald MN, Cho MH, Hobbs BD, Parker MM, DeMeo DL, Hersh $\mathrm{CP}$, Ehringer MA et al: Common and Rare Variants Genetic Association Analysis of Cigarettes per Day Among Ever-Smokers in Chronic Obstructive Pulmonary Disease Cases and Controls. Nicotine \& tobacco research : official journal of the Society for Research on Nicotine and Tobacco 2019, 21(6):714-722.

15. Perera MA: The missing linkage: what pharmacogenetic associations are left to find in CYP3A? Expert opinion on drug metabolism \& toxicology 2010, 6(1):17-28.

16. Kedmi M, Maayan S, Cohen SB, Hauzi M, Rund D: MDR1 and CYP3A4 polymorphisms are associated with HIV seropositivity in Israeli patients but do not influence the course of HIV disease. AIDS patient care and STDs 2007, 21(9):653-658.

17. Tayeb MT, Clark C, Sharp L, Haites NE, Rooney PH, Murray GI, Payne SN, McLeod HL: CYP3A4 promoter variant is associated with prostate cancer risk in men with benign prostate hyperplasia. Oncology reports 2002, 9(3):653-655.

18. Yamada Y, Matsuo H, Watanabe S, Kato K, Yajima K, Hibino T, Yokoi K, Ichihara S, Metoki N, Yoshida $\mathrm{H}$ et al: Association of a polymorphism of CYP3A4 with type 2 diabetes mellitus. International journal of molecular medicine 2007, 20(5):703-707.

19. Kang YS, Park SY, Yim CH, Kwak HS, Gajendrarao P, Krishnamoorthy N, Yun SC, Lee KW, Han KO: The CYP3A4*18 genotype in the cytochrome P450 3A4 gene, a rapid metabolizer of sex steroids, is associated with low bone mineral density. Clinical pharmacology and therapeutics 2009, 85(3):312318.

20. Roberts JK: Metabolism of inhaled glucocorticoids and CYP3A gene regulation in lung cells. Dissertations \& Theses - Gradworks 2013.

21. Zanger UM, Schwab M: Cytochrome P450 enzymes in drug metabolism: regulation of gene expression, enzyme activities, and impact of genetic variation. Pharmacology \& therapeutics 2013, 138(1):103-141.

22. Ohtsuki S, Schaefer O, Kawakami H, Inoue T, Liehner S, Saito A, Ishiguro N, Kishimoto W, LudwigSchwellinger E, Ebner T et al: Simultaneous absolute protein quantification of transporters, cytochromes P450, and UDP-glucuronosyltransferases as a novel approach for the characterization of individual human liver: comparison with mRNA levels and activities. Drug metabolism and disposition: the biological fate of chemicals 2012, 40(1):83-92.

23. Hukkanen J, Pelkonen $\mathrm{O}$, Hakkola J, Raunio H: Expression and regulation of xenobiotic-metabolizing cytochrome P450 (CYP) enzymes in human lung. Critical reviews in toxicology 2002, 32(5):391-411.

24. Tesfaigzi Y, Kluger M, Kozak W: Clinical and cellular effects of cytochrome P-450 modulators. Respiration physiology 2001, 128(1):79-87.

25. Giembycz MA, Field SK: Roflumilast: first phosphodiesterase 4 inhibitor approved for treatment of COPD. Drug design, development and therapy 2010, 4:147-158. 
26. Shimada T, Martin MV, Pruess-Schwartz D, Marnett LJ, Guengerich FP: Roles of individual human cytochrome P-450 enzymes in the bioactivation of benzo(a)pyrene, 7,8-dihydroxy-7,8dihydrobenzo(a)pyrene, and other dihydrodiol derivatives of polycyclic aromatic hydrocarbons. Cancer research 1989, 49(22):6304-6312.

27. Piipari R, Savela K, Nurminen T, Hukkanen J, Raunio H, Hakkola J, Mantyla T, Beaune P, Edwards RJ, Boobis AR et al: Expression of CYP1A1, CYP1B1 and CYP3A, and polycyclic aromatic hydrocarbonDNA adduct formation in bronchoalveolar macrophages of smokers and non-smokers. International journal of cancer 2000, 86(5):610-616.

28. Roberts-Thomson SJ, McManus ME, Tukey RH, Gonzalez FF, Holder GM: The catalytic activity of four expressed human cytochrome P450s towards benzo[a]pyrene and the isomers of its proximate carcinogen. Biochemical and biophysical research communications 1993, 192(3):1373-1379.

29. Amirimani B, Ning B, Deitz AC, Weber BL, Kadlubar FF, Rebbeck TR: Increased transcriptional activity of the CYP3A4*1B promoter variant. Environmental and molecular mutagenesis 2003, 42(4):299305.

30. Hu YF, He J, Chen GL, Wang D, Liu ZQ, Zhang C, Duan LF, Zhou HH: CYP3A5*3 and CYP3A4*18 single nucleotide polymorphisms in a Chinese population. Clinica chimica acta; international journal of clinical chemistry 2005, 353(1-2):187-192. 